\title{
Efeitos da queima controlada sobre algumas propriedades químicas do solo em um povoamento de Pinus taeda no município de Sengés - PR
}

\author{
Antonio Carlos Batista* \\ Carlos Bruno Reissmann* \\ Ronaldo Viana SoARES**
}

\begin{abstract}
RESUMO
Este trabalho teve por objetivo avaliar o efeito de uma queima controlada sobre as propriedades químicas do solo de um povoamento de Pinus taeda. Para obtenção dos dados foram alocados aleatoriamente 5 pontos amostrais em uma área de $1000 \mathrm{~m}^{2}$. Em cada um destes pontos foram estabelecidas 4 amostras, 2 a serem queimadas e 2 sem queimar. Após a queima foram abertos micro-perfis em cada amostra, coletando-se material da serapilheira recém-caida (L) e semi-decomposta (F) e de solo nas profundidades de 0 a $5 \mathrm{~cm}, 5$ a $10 \mathrm{~cm}, 10$ a $15 \mathrm{~cm}$ e 15 a $20 \mathrm{~cm}$. Os resultados mostraram uma redução (não significativa) de $\mathrm{N}$ e $\mathrm{K}$ e um aumento (não significativo) de $\mathrm{Ca}$ e $\mathrm{Mg}$ na serapilheira das áreas queimadas. A análise do $\mathrm{pH}$ do solo mostrou não haver diferença significativa entre áreas queimadas e não queimadas. Houve um aumento também não significativo da concentração de $\mathrm{Ca}, \mathrm{Mg}, \mathrm{K}$ e $\mathrm{P}$ nas camadas superficiais do solo após a queima.
\end{abstract}

Palavras-chave: queima controlada, efeitos do fogo, Pinus taeda

\begin{abstract}
Effects of prescribed burning on soil chemical properties in a loblolly pine plantation in Sengés - PR, Brazil. The objective of this research was to evaluate the effects of a prescribed burning on soil chemical properties in a loblolly pine plantation. Data were collected from 5 random sites, in a 1,000 $\mathrm{m}^{2}$ área. From each site 4 plots, two burned and two unburned, were demarcated. After the burn was made, profiles were open in each plot and samples of fresh litter, partially decomposed litter, and soil at $0-5 \mathrm{~cm}, 5$ $10 \mathrm{~cm}, 10-15 \mathrm{~cm}$, and $15-20 \mathrm{~cm}$ dephts were collected. Results showed a statistically non significative reduction of $\mathrm{N}$ and $\mathrm{K}$, and an also non significative increase of $\mathrm{Ca}$ and $\mathrm{Mg}$ in the litter layer of the burned plots. Analysis of soil $\mathrm{pH}$ presented no significative difference
\end{abstract}

\footnotetext{
* Engenheiro Florestal, M.Sc.; Dr., professor adjunto da UFPR.

** Engenheiro Florestal, M.Sc., Ph.D., professor titular da UFPR.
} 
between burned and unburned plots. It was also observed a non significative increase on the levels of $\mathrm{Ca}, \mathrm{Mg}, \mathrm{K}$ and $\mathrm{P}$ in the soil superficial layers.

Key words: prescribed burning, fire effects, Pinus taeda

\section{INTRODUÇÃO}

Os incêndios florestais são uma ameaça constante e o risco e a intensidade de danos aumenta, dentre outros aspectos, em função da extensão da área, da idade dos povoamentos e da intensidade do fogo.

$\mathrm{O}$ acúmulo de material combustível sob povoamentos de pinus ao longo dos anos aumenta drasticamente o risco de incêndios. Uma das alternativas para diminuir este risco ou diminuir o potencial de danos dos incêndios é reduzir periodicamente a quantidade de material combustível no interior dos povoamentos através de queima controlada.

Um dos principais questionamentos relacionados ao uso da queima controlada em povoamentos florestais é sobre os efeitos do fogo no solo. Em razão disto, este trabalho teve por objetivo avaliar os efeitos da queima controlada sobre as propriedades químicas do solo, em uma plantação de Pinus taeda.

\section{REVISÃO DA LITERATURA}

De uma maneira simplificada pode-se dizer que os efeitos diretos da combustão no ecossistema são o consumo da matéria orgânica, a produção de calor (pela conversão de energia química em energia calórica) e a liberação de nutrientes e de sub-produtos da combustão. Como conseqüência desses efeitos diretos, os reflexos ou efeitos secundários serão observados em maior ou menor escala, dependendo da intensidade do fogo, nos diversos elementos do ecossistema, tais como: o solo e a vegetação (MARTIN et al., 1977).

O fogo converte a biomassa sólida em gases e vapor d'água e ao mesmo tempo libera grandes quantidades de energia, deixando no solo as cinzas com alta concentração de nutrientes. Portanto, o fogo afeta o solo de três maneiras:

a) pela exposição, deixando-o descoberto;

b) pelo aquecimento;

c) pela liberação de nutrientes.

O grau de aquecimento do solo durante uma determinada queima é altamente variável e depende do tipo de combustível (herbáceas, arbustos, árvores), da intensidade do fogo (incêndio, queima controlada), da natureza da camada de serapilheira (espessura, conteúdo de umidade), e das propriedades do solo (matéria orgânica, teor de umidade, textura). Diversas características do solo, tais como estrutura, capacidade de troca de cátions, disponibilidade de nutrientes e atividade microbiológica, são altamente dependentes da matéria orgânica, que começa a se alterar quimicamente quando aquecida a $200{ }^{\circ} \mathrm{C}$ e é completamente consumida quando atinge $450{ }^{\circ} \mathrm{C}(\mathrm{DE} B A N O, 1989)$. 
REGO et al. (1983), estudando os efeitos da queima controlada em povoamentos de Pinus pinaster em Portugal, verificaram uma redução acentuada da matéria orgânica após a passagem do fogo, nas áreas analisadas. Segundo os autores, a variação da matéria orgânica é muito importante devido as consequências sobre as outras características do solo e também sobre a fauna do solo.

KUTIEL \& NAVEH (1987), pesquisando sobre as propriedades do solo sob um povoamento misto de Pinus halepensis e Quercus calliprinos, em áreas queimadas e não queimadas, verificaram uma redução de cerca de $50 \%$ da matéria orgânica do solo, tanto sob Pinus como sob Quercus. Já AUSTIN \& BAISINGER (1955), analisando os efeitos da queima controlada em povoamentos de Pseudotsuga menziesii, verificaram uma redução de 75,5\% da matéria orgânica do solo pelo fogo.

MARTIN (1981) afirma que cerca de 60 a $80 \%$ do nitrogênio existente no material combustível consumido pelo fogo é perdido para a atmosfera, mas geralmente aumenta a quantidade de nitrogênio sob a forma disponível para as plantas. SCHOCH \& BINKLEY (1986) afirmam que as perdas de nitrogênio nas queimas controladas de média e alta intensidade em povoamentos de diversas espécies de pinus varia de 50 a $200 \mathrm{~kg} \cdot \mathrm{ha}^{-1}$.

DE BANO (1989) afirma que grandes quantidades de alguns nutrientes, tais como $\mathrm{N}, \mathrm{S}$ e $\mathrm{P}$ podem ser volatilizados durante um incêndio. Cátions, tais como $\mathrm{Ca}, \mathrm{Mg}, \mathrm{K}$ e $\mathrm{Na}$, não são volatilizados, embora pequenas quantidades possam ser transferidas do sítio pela fumaça.

SOARES (1990) analisou os efeitos da queima controlada em povoamentos de Pinus caribaea e $P$. oocarpa. Os resultados indicaram que a concentração do $\mathrm{N}$ na serapilheira foi reduzida em 44 e 39,2\% e o P de 45 e 41,6\%, respectivamente. Os outros elementos analisados (K, $\mathrm{Ca}, \mathrm{Mg}, \mathrm{Fe}, \mathrm{Mn}, \mathrm{Cu}$ e B) aumentaram suas concentrações após a queima, exceto o Ca no povoamento de $P$. caribaea. Todos os elementos disponíveis (mineralizados) aumentaram significativamente na camada superior do solo, após o fogo, retornando aos níveis anteriores após 7 meses.

VEGA et al. (1983) analisaram a variação da quantidade de nutrientes em áreas experimentais queimadas e não queimadas de Pinus pinaster e Pinus radiata. A análise dos dados mostrou que não houve grandes modificações no solo mineral devido as baixas intensidades do fogo e também porque os experimentos foram realizados numa estação em que o conteúdo de água nos combustíveis e no solo era relativamente alta. Houve uma queda na quantidade total de $\mathrm{N}$ e de matéria orgânica e um leve aumento nas concentrações de $\mathrm{Ca}, \mathrm{Mg}, \mathrm{Ke}$ P, oito meses após a queima, embora não tenha sido verificada diferença significativa. Segundo os autores o pequeno aumento verificado foi devido a liberação de bases da vegetação e do material morto queimados.

MAAGS (1988) avaliou alguns efeitos da queima controlada em plantações de Pinus elliottii no sudoeste de Queensland, Austrália. Os resultados demonstraram que uma única queima controlada reduziu até $52 \%$ da biomassa total. A biomassa e as quantidades de $\mathrm{N}, \mathrm{P}, \mathrm{Na}$ e $\mathrm{Mg}$ foram significativamente 
menores no piso florestal queimado até 1,5-2,5 anos após a queima, mas as diferenças declinaram rapidamente, e após 3 anos nenhum efeito da queima ficou evidente. As quantidades de $\mathrm{P}$ e Ca não diferiram significativamente entre os tratamentos exceto para o Ca na primeira coleta após o fogo. As concentrações de $\mathrm{Ca}$ foram significativamente mais altas no piso florestal queimado até 1,5 ano após a queima, sugerindo que uma proporção significante de $\mathrm{Ca}$ na serapilheira queimada foi liberado vagarosamente, comparado com outros nutrientes.

Quando a matéria orgânica do solo é queimada, muitas das substâncias nela contidas são liberadas em forma de óxidos, remanescendo os carbonatos, que geralmente apresentam reação alcalina. Desse modo, quando as cinzas são depositadas sobre o solo, a tendência é diminuir a acidez. SOARES (1985) relata experimentos de queimas controladas onde a acidez foi reduzida em 2 a 3 unidades de $\mathrm{pH}$, voltando ao normal 5 anos após a queima.

De acordo com WELLS et al. (1979), a acidez nas camadas superficiais do solo é reduzida pela queima, como resultado dos cátions básicos liberados pela combustão da matéria orgânica e efeitos químicos do aquecimento sobre a matéria orgânica e os minerais . $\mathrm{O}$ pH do solo é elevado temporariamente dependendo da quantidade de cinzas liberada, do pH original do solo, da composicão da cinza e da umidade do local.

\section{MATERIAL E MÉTODOS}

O experimento foi realizado em uma plantação de Pinus taeda L. de propriedade da PISA Florestal S.A., localizada no município de Sengés-Paraná, situada a $24^{\circ} 10^{\prime}$ de latitude sul e $49^{\circ} 35^{\prime}$ de longitude oeste de Greenwich, distante aproximadamente $280 \mathrm{~km}$ da cidade de Curitiba. A altitude média é de 960 metros s.n.m.

Segundo a classificação de Köppen o clima é caracterizado como Cfa , sempre úmido, com o mês mais quente apresentando temperatura média superior a $22^{\circ} \mathrm{C}$, doze meses com temperatura superior a $10^{\circ} \mathrm{C}$ e com mais de cinco geadas por ano (MAACK, 1968). A temperatura média anual é de $18,5 \mathrm{C}$ e a precipitação média anual é de $1720 \mathrm{~mm}$ (média de 20 anos). Junho e julho são os meses que apresentam a menor precipitação média.

De acordo com MAACK (1968), a predominância da direção dos ventos é dos quadrantes sulinos, com $38.1 \%$, que segundo o autor são os ventos que desfazem as chuvas. Os ventos que sopram dos quadrantes norte perfazem $29.2 \%$ e são os causadores de chuva. Há também $23.2 \%$ de calmarias.

Geologicamente a área compreende arenitos do Grupo Guatá.

A queima foi realizada no ano de 1993, no dia 5 de novembro, após 10 dias sem chuva. O sumário das condições meteorológicas do comportamento do fogo durante a queima está apresentado na Tabela 1 . 
Tabela 1. Condições meteorológicas e comportamento do fogo durante a queima

Table 1. Meteorological conditions and fire behavior during the prescribed burning

Hora da queima : 10:50 a 11:15 Combustível consumido : 0,383 kg.m-2

Temperatura do ar: $25^{\circ} \mathrm{C}$

Vel. De propagação do fogo: $0,013 \mathrm{~m} \cdot \mathrm{s}^{-1}$

Umidade relativa do ar: $51 \%$

Intensidade do fogo: $21,2 \mathrm{kcal} . \mathrm{m} . \mathrm{s}^{-1}$

Velocidade do vento: $0,5 \mathrm{~m} . \mathrm{s}^{-1} \quad$ Calor liberado: $1630,7 \mathrm{kcal} . \mathrm{m}^{-2}$

Peso mat. Combustível total: 1,38 kg.m-2

Para caracterização do solo da área do experimento, foi aberta uma trincheira para análise do perfil sendo o solo classificado pelo Professor Dr. Arthur Santos Filho (SCA/UFPR) ${ }^{2}$ como do tipo Cambissolo Tb álico A moderado, textura média substrato arenito.

A área do experimento,de acordo com o sistema fitofisionômicoecológico proposto pelo IBGE (1990), enquadra-se na região fitogeográfica de Savana Arbórea Aberta. Esta tipologia concentra-se principalmente nas proximidades de Itararé (SP) e Jaguariaiva e Tibagi (PR).

O experimento foi feito em uma área de Pinus taeda do talhão 38 do projeto Mocambo VII, entre as cotas 860 e $830 \mathrm{~m}$ acima do nível do mar, com uma inclinação de aproximadamente $20 \%$.

O plantio foi efetuado em 1977/1978, com espaçamento inicial de 2 X 2,5 m. No ano de 1991 o povoamento se encontrava com 13 anos, não tinha sofrido nenhum desbaste e apresentava altura média de 15,5 m e DAP médio de 19,5 cm.

Para comprovação da espécie Pinus taeda foram coletadas amostras de material fértil, as quais foram encaminhadas ao laboratório de Dendrologia do Departamento de Silvicultura e Manejo da Universidade Federal do Paraná para identificação. A duplicata do material encontra-se catalogada e arquivada no Herbário "Escola de Florestas Curitiba" (EFC) sob registro N $^{\circ} 3777$.

Para avaliação dos efeitos sobre o solo foram estabelecidos 5 pontos amostrais ao acaso numa parcela de $1000 \mathrm{~m} 2(20 \times 50 \mathrm{~m})$. Em cada um destes pontos foram estabelecidas quatro amostras: duas a serem queimadas e duas sem queimar.

Vinte e quatro horas após a passagem do fogo foram abertos microperfis no solo, em cada amostra. Em cada microperfil, o material foi coletado nas seguintes camadas de solo:

- serapilheira recém-caida $(\mathrm{L})$;

- serapilheira semi-decomposta $(\mathrm{F})$;

- Camada de solo da profundidade de 0 a $5 \mathrm{~cm}$;

- Camada de solo da profundidade de 5 a $10 \mathrm{~cm}$;

2 Comunicação pessoal 
- Camada de solo da profundidade de 10 a $15 \mathrm{~cm}$;

- Camada de solo da profundidade de 15 a $20 \mathrm{~cm}$.

O material da serapilheira foi analisado no laboratório de nutrição de plantas e as amostras dos horizontes do solo no laboratório de química e fertilidade do solo do Departamento de Solos da Universidade Federal do Paraná.

Na serapilheira foram analisados nitrogênio $(\mathrm{N})$, potássio $(\mathrm{K})$, cálcio $(\mathrm{Ca})$ e magnésio $(\mathrm{Mg})$, de acordo com metodologia proposta por HILDEBRAND (1976).

Para as amostras de solo procedeu-se uma análise química de rotina (EMBRAPA, 1979).

Para a análise estatística utilizou-se o delineamento totalmente casualizado. Os testes empregados foram a análise de variância (ANOVA), teste de comparação de médias SNK para mais de duas médias e teste "T" para comparação de duas médias.

O nível de significância utilizado nos testes estatísticos foi de $95 \%$.

\section{RESULTADOS E DISCUSSÃO}

Os conteúdos totais dos elementos analisados na serapilheira, antes e após a queima de 1993, são apresentados na tabela 2. A análise de variância não mostrou diferença significativa entre os teores de nutrientes das parcelas queimadas e não queimadas. Embora não significativos, pode-se observar que os valores de $\mathrm{N}$ e $\mathrm{K}$ foram menores nas parcelas queimadas, enquanto que as parcelas queimadas apresentaram maiores teores de $\mathrm{Ca} \mathrm{e} \mathrm{Mg}$.

Pelos resultados encontrados pode-se afirmar que não houve alterações significativas nos teores de $\mathrm{N}, \mathrm{K}, \mathrm{Ca}$ e $\mathrm{Mg}$ da serapilheira. Os resultados encontrados por SOARES (1990) e MAGGS (1988), em trabalhos semelhantes indicam uma redução de $\mathrm{N}$ na serapilheira das parcelas queimadas e um aumento de $\mathrm{K}, \mathrm{Ca}$ e Mg.

Tabela 2. Conteúdos totais de $\mathrm{N}, \mathrm{K}, \mathrm{Ca}$ e $\mathrm{Mg}$ encontrados na serapilheira taeda, nas parcelas queimadas e não queimadas

Tabela 2. Total content of $\mathrm{N}, \mathrm{K}, \mathrm{Ca}$, and $\mathrm{Mg}$ found in the litter of the burned and unburned plots

\begin{tabular}{|c|c|c|c|c|}
\hline \multirow[b]{2}{*}{ Tratamento } & \multicolumn{4}{|c|}{ 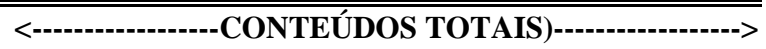 } \\
\hline & $N\left(g .100 g^{-1}\right)$ & $\mathrm{K}\left(\mathbf{m g} \cdot \mathrm{kg}^{-1}\right)$ & $\mathrm{Ca}\left(\mathrm{mg} . \mathrm{kg}^{-1}\right)$ & $\operatorname{Mg}\left(\mathbf{m g} . \mathrm{kg}^{-1}\right)$ \\
\hline Queimado & 0,63 & 205,9 & 1330 & 214 \\
\hline Não Queimado & 0,67 & 279,7 & 920 & 163 \\
\hline
\end{tabular}

Pares de médias na coluna seguidas de "*" diferem entre si pelo Teste de T ao nível de 5\%.

Means in the columns, followed by * are significantly different by the T test at .05 level. 
A análise de variância das características relacionadas com a acidez do solo não mostrou diferença significativa entre as parcelas queimadas e não queimadas (tabela 3). Os valores de $\mathrm{Al}^{+3} \mathrm{e} \mathrm{H}+\mathrm{Al}$ foram menores nas áreas não queimadas, embora tenha ocorrido um pequeno incremento na profundidade de 0 a $5 \mathrm{~cm}$. SOARES (1990), obteve um aumento não significativo no $\mathrm{pH}$ de 4,21 para 4,33 em camadas de solo de até $2,5 \mathrm{~cm}$ de profundidade em áreas queimadas sob Pinus caribaea e um aumento significativo de 3,98 para 4,13 nas áreas queimadas de P. oocarpa em Sacramento, Minas Gerais. VEGA et al. (1983) observaram também uma pequena diferença não significativa entre o $\mathrm{pH}$ de áreas não queimadas $(\mathrm{pH}=4,52)$ e áreas queimadas $(\mathrm{pH}=4,60)$. DE RONDE et al. $(1990)$ afirmam que o efeito do fogo no $\mathrm{pH}$ decresce com a profundidade do solo. Os valores de $\mathrm{pH}$ nas profundidades analisadas não permitiram uma clara definição. No entanto a maior diferença se deu na camada mais superficial do solo.

Tabela 3. Valores de $\mathrm{pH}$ e teores de $\mathrm{Al}^{+3}$ e $\mathrm{H}+\mathrm{Al}$ nas camadas do solo de áreas queimadas e não queimadas

Table 3. $\mathrm{pH}$ values and $\mathrm{Al}^{+3}$ and $\mathrm{H}+\mathrm{Al}$ contents in the soil layers of burned and unburned plots

\begin{tabular}{|c|c|c|c|c|}
\hline \multirow[t]{2}{*}{ TRATAMENTO } & \multicolumn{4}{|c|}{ PROFUNDIDADE } \\
\hline & $0-5 \mathrm{~cm}$ & $5-10 \mathrm{~cm}$ & $10-15 \mathrm{~cm}$ & $15-20 \mathrm{~cm}$ \\
\hline & \multicolumn{4}{|c|}{$\mathrm{pH}\left(\mathrm{CaCl}_{2}\right)$} \\
\hline Queimada & 3,86 & 3,98 & 3,98 & 4,06 \\
\hline \multirow[t]{2}{*}{ Não queimada } & 3,81 & 3,98 & 4,02 & 4,04 \\
\hline & \multicolumn{4}{|c|}{$\mathrm{Al}^{+3}\left(\mathrm{cmol}_{\mathrm{c}} \cdot \mathrm{kg}^{-1}\right)$} \\
\hline Queimada & 1,78 & 1,58 & 1,46 & $1,28 *$ \\
\hline \multirow[t]{2}{*}{ Não queimada } & 1,91 & 1,55 & 1,46 & $1,35 *$ \\
\hline & \multicolumn{4}{|c|}{$\mathrm{H}+\mathrm{Al}\left(\mathrm{cmol}_{\mathrm{c}} \cdot \mathrm{kg}^{-1}\right)$} \\
\hline Queimada & 8,80 & 7,78 & 7,36 & 6,68 \\
\hline Não queimada & 8,93 & 7,58 & 7,17 & 6,80 \\
\hline
\end{tabular}

Pares de médias seguidas de "*"diferem entre si pelo Teste T ao nível de $5 \%$.

Means, in the columns, followed by * are significantly different by the T test at .05 level.

Na tabela 4 estão apresentados os valores médios dos teores de matéria orgânica (M.O), capacidade de troca catiônica (CTC), saturação de Alumínio $(\mathrm{m} \%)$ e saturação de bases (V\%), nas camadas superficiais do solo de áreas queimadas e não queimadas.

Os resultados da tabela 4 mostram que a queima não provocou alteração estatisticamente significativa em nenhuma das características analisadas.

Para a matéria orgânica, os resultados indicam que não houve redução pela queima. HUNT \& SIMPSON (1985) encontraram resultados semelhantes quando analisaram solos de áreas queimadas de povoamentos de Pinus elliottii. McKEE (1982) observou um aumento do teor de matéria orgânica em solos de áreas queimadas de povoamentos de Pinus palustris, $P$. elliottii e P. taeda. No entanto, em muitos outros experimentos, os resultados indicaram redução do teor de matéria orgânica do solo devido a queima (KUTIEL \& NAVEH, 1987; REGO 
et al., 1983; VEGA et al., 1983, AUSTIN \& BAISINGER, 1955). De modo geral, a intensidade do fogo e as características do solo são os fatores mais importantes que concorrem para redução da matéria orgânica pela queima.

Ao se analisar a CTC, observa-se comportamento semelhante ao ocorrido com a matéria orgânica.

Tabela 4. Teores de matéria orgânica (M.O.), capacidade de troca de cátions (CTC), saturação de alumínio (M) e saturação de bases (V) em camadas superficiais de solos de áreas queimadas e não queimadas

Table 4. Organic matter $(M O)$, cation exchange capacity $(C T C)$, aluminum saturation $(M)$, and bases saturation $(V)$ in the soil layers of burned and unburned plots

\begin{tabular}{|c|c|c|c|c|}
\hline \multirow[t]{2}{*}{ TRATAMENTO } & \multicolumn{4}{|c|}{ PROFUNDIDADE } \\
\hline & $0-5 \mathrm{~cm}$ & $5-10 \mathrm{~cm}$ & $10-15 \mathrm{~cm}$ & $15-20 \mathrm{~cm}$ \\
\hline & \multicolumn{4}{|c|}{ M.O. \% } \\
\hline Queimada & 3.5 & 2.3 & 2.1 & 1.4 \\
\hline \multirow[t]{2}{*}{ Não queimada } & 3.6 & 2.4 & 1.6 & 1.3 \\
\hline & \multicolumn{4}{|c|}{ СТC \% } \\
\hline Queimada & 9.87 & 8.72 & 8.20 & 7.39 \\
\hline \multirow[t]{2}{*}{ Não queimada } & 9.96 & 8.45 & 7.87 & 7.47 \\
\hline & \multicolumn{4}{|c|}{$\mathrm{m} \%$} \\
\hline Queimada & 62.6 & 63.0 & 64.2 & 64.2 \\
\hline \multirow[t]{2}{*}{ Não queimada } & 65.2 & 64.2 & 68.1 & 67.0 \\
\hline & \multicolumn{4}{|c|}{$\mathrm{V} \%$} \\
\hline Queimada & 11.0 & 10.8 & 10.1 & 9.8 \\
\hline Não queimada & 10.3 & 10.3 & 8.7 & 9.1 \\
\hline
\end{tabular}

Pares de médias seguidas de "*" diferem entre si pelo Teste $\mathrm{T}$ ao nível de $5 \%$.

Means, in the columns, followed by * significantly different by the T test at .05 level.

Os resultados da tabela 5 indicam não haver diferença significativa entre os teores dos cátions nas áreas queimadas e não queimadas. Pode-se observar também que há uma tendência, não testada, de redução gradual dos teores dos cátions a medida que se afasta da superfície. 
Tabela 5. Teores de cátions trocáveis $\mathrm{Ca}^{+2}, \mathrm{Mg}^{+2}, \mathrm{~K}^{+}$e $\mathrm{P}$ nas camadas superficiais do solo de áreas queimadas e não queimadas

Table 5. Available $\mathrm{Ca}^{+2}, \mathrm{Mg}^{+2}, \mathrm{~K}^{+}$, and $\mathrm{P}$ in the soil layers of burned and unburned plots

\begin{tabular}{|c|c|c|c|c|}
\hline \multirow[t]{2}{*}{ TRATAMENTO } & \multicolumn{4}{|c|}{$\begin{array}{ll}\text { PROFUNDIDADE } \\
\end{array}$} \\
\hline & $0-5 \mathrm{~cm}$ & $5-10 \mathrm{~cm}$ & $10-15 \mathrm{~cm}$ & $15-20 \mathrm{~cm}$ \\
\hline & \multicolumn{4}{|c|}{$\mathrm{Ca}^{+2}\left(\mathrm{cmol}_{\left.\mathrm{c} \cdot \mathrm{kg}^{-1}\right)}\right.$} \\
\hline Queimada & 0.6 & 0.6 & 0.5 & 0.4 \\
\hline \multirow[t]{2}{*}{ Não queimada } & 0.6 & 0.5 & 0.4 & 0.4 \\
\hline & \multicolumn{4}{|c|}{$\mathrm{Mg}^{+2}\left(\mathrm{cmol}_{\mathrm{c}} \cdot \mathrm{kg}^{-1}\right)$} \\
\hline Queimada & 0.4 & 0.3 & 0.3 & 0.2 \\
\hline \multirow[t]{2}{*}{ Não queimada } & 0.3 & 0.3 & 0.2 & 0.2 \\
\hline & \multicolumn{4}{|c|}{$\mathrm{K}^{+}\left(\mathrm{cmol}_{\left.\mathrm{c} \cdot \mathrm{kg}^{-1}\right)}\right.$} \\
\hline Queimada & 0.10 & 0.07 & 0.06 & 0.04 \\
\hline \multirow[t]{2}{*}{ Não queimada } & 0.09 & 0.07 & 0.05 & 0.04 \\
\hline & \multicolumn{4}{|c|}{$P\left(\mathrm{mg} \mathrm{kg}^{-1}\right)$} \\
\hline Queimada & 3.9 & 2.5 & 1.9 & 1.3 \\
\hline Não queimada & 3.1 & 2.6 & 2.0 & 1.2 \\
\hline
\end{tabular}

Pares de médias seguidas de "*" diferem entre si pelo teste t ao nível de $5 \%$.

Means, in the columns, followed by * are significantly different by T test at .05 level.

Embora não tenha sido detectada diferença significativa, todos os elementos analisados apresentam teores levemente maiores nas parcelas queimadas. Resultados similares têm sido encontrados por diversos pesquisadores. VEGA et al., (1983) analisando a variação da quantidade de nutrientes em função da queima em povoamentos de $P$. pinaster e $P$. radiata na Espanha, verificaram um pequeno aumento nas concentrações de $\mathrm{Ca}, \mathrm{Mg}, \mathrm{K}$ e $\mathrm{P}$ nas parcelas queimadas, embora não significativo estatisticamente.

Também é preciso considerar que o solo era naturalmente pobre em bases trocáveis, o que por sua vez também gerou um litter mais pobre em elementos desta categoria. Alia-se a isto o fato do Pinus taeda ser uma das espécies menos exigentes em Ca (LUTZ \& CHANDLER 1947). Desta forma, apesar do Ca ser um elemento imóvel nos tecidos, e portanto, acumular nos órgãos mais velhos, sua contribuição no caso do litter de $P$. taeda foi pouco expressiva. O seu nível encontrado no litter não queimado ( $920 \mu \mathrm{g} . \mathrm{g}^{-1}$ M.S.) ficou bastante aquém do encontrado no litter de povoamentos de regiões das mais pobres do Paraná (2000 $\mu \mathrm{g} . \mathrm{g}^{-1}$ M.S.) (REISSMANN, 1981). Para o Mg, outro elemento que se somaria às bases trocáveis, também se verificou um teor baixo, compatível com o encontrado em solos pobres, desenvolvidos em regiões de arenitos. Já a contribuição do K foi irrisória. Naturalmente pela baixa fertilidade do solo, agravado pela alta taxa de redistribuição interna na árvore, que deve ter sido intensificada para o seu deslocamento às acículas remanescentes na copa.

Em um estudo realizado por KUTIEL \& NAVEH (1987), em povoamentos de Pinus halepensis e Quercus calliprinos em Israel, verificou-se 
um aumento significativo de K e Mg nas áreas queimadas. McKEVLIN \& McKEE ( 1986) analisando as propriedades químicas dos solos de áreas queimadas de Pinus taeda nos EUA, observaram uma maior quantidade de $\mathrm{P}, \mathrm{K}, \mathrm{Ca}$ e $\mathrm{Mg}$ nos solos queimados. HUNT \& SIMPSON (1985) analisaram os efeitos de queimas de baixa intensidade no crescimento e nutrição das árvores de um povoamento de Pinus elliottii em Queensland. Os resultados mostraram uma maior quantidade K, $\mathrm{Ca}$ e $\mathrm{Mg}$ nas parcelas queimadas.

\section{CONCLUSÕES}

Os resultados obtidos neste experimento permitem concluir que:

A análise do teor de nutrientes da serapilheira antes e depois da queima não mostrou alteração significativa.

Não houve alteração significativa na acidez do solo devido a queima.

Não foi detectada alteração significativa dos elementos $\mathrm{Ca}^{+2}, \mathrm{Mg}^{+2}, \mathrm{~K}^{+}$ e P nas camadas superficiais do solo, após a queima.

\section{BIBLIOGRAFIA CITADA}

AUSTIN, R.C.; BAISINGER, D.H. Some effects of burning on forest soils of western Oregon and Washington. Journal of Forestry, Washington, n.53, p.275-280, 1955.

DE BANO, L.F. Effects of fire on chaparral soils in Arizona and California and postfire management implications. In: Symposium on Fire and Watershed Management (1988: Sacramento). Proceedings. Gen Tech. Rep., U.S.D.A. Forest Service, Berkeley, PSW-109, 1989, p.55-62.

DE RONDE, C.;GOLDAMMER, J.G.;WADE, D.D.; SOARES, R.V. Prescribed fire in industrial plantations. In: GOLDAMMER, J.G. (ed.). Fire in the Tropical Biota Ecosystem Processes and Global Challenges. Berlin: Springer-Verlag, 1990, p.216272, (Ecological Studies, Vol.84).

EMBRAPA. Manual de métodos de análise de solo. Rio de Janeiro: Ministério da Agricultura, 1979.

HILDEBRAND, C. Manual de análise química de solo e de plantas. Curitiba: UFPR, 1976. (mimeografado).

HUNT, S.M.; SIMPSON, J.A. Effects of low intensity prescribed fire on the growth and nutrition of slash pine plantation. Aust. For. Res. , v.15, p.67-77, 1985.

IBGE. Geografia do Brasil: Região Sul, v.2. Rio de Janeiro, 420p, 1990. 
KUTIEL, P.; NAVEH, Z. Soil properties beneath Pinus halepensis and Quercus calliprinos trees on burned and unburned mixed forest on Mt. Carmel, Israel. For. Ecol. and Manag., Amsterdam, v.20, p.11-24, 1987.

LUTZ, H.J.; CHANDLER, R.F. Jr. Forest Soils. John Wiley \& Sons. New York. 514p. 1947.

MAACK, R. Geografia física do estado do Paraná Curitiba: BADEP/UFPR/IBPT, 350p, 1968.

MAGGS, J. Organic matter and nutrients in the forest floor of a Pinus elliotti plantation and some effects of prescribed burning and superphosphate addition. For. Ecol. and Manag., Amsterdam, v.23, p.105-109, 1988.

MARTIN, R.E. Precribed burning techniques to maintain or improve soil productivity. In: HOBS, S.D. and HELGERSON, O.T. (Eds.). Reforestation of skeletal soils: Proceedings of a workshop; 1981 November 17-19; Medford, OR. Corvallis, OR.: Forest Research Laboratory, Oregon State University; 1981, p.66-70.

MARTIN , R.E; COOPER, R.W.; CROW, A.B.; CUMMING, J.A. and PHILIPS, C.B. Report of task force on prescribed burning. Journal of Forestry, Washington, v.75, n.5, p.297-301, 1977

McKEE, W.H. Jr. Changes in soil fertility following prescribed burning on Coastal Plain pine sites. Res. Pap., Asheville, U.S.D.A. Forest Service, SE-234, 23p., 1982.

McKEVLIN ; M.R.; McKEE, W.H.Jr. Long term prescribed burning increases nutrient uptake and growth of lobboly pine seedlings. For. Ecol. Manag. Amsterdam, v.17, p.245-252, 1986.

REGO, F.G.; SILVA, J.M.; CABRAL, M.T. The use of prescribed burning in the Nortwest of Portugal. In: GOLDAMMER,J.G.(HRSg). DFG - Symposium "Feuerökologie". Albert Ludwigs - Universitat Freiburg i.Br. p.88-104, 1983.

REISSMANN, C.B. Nährelementversorgung und Wuchsleistung von kiefernbeständen in Südbrasilien. Tese Doutorado - Universität Albert Ludwig Freiburg i. Br. Alemanha. 159p. 159p

SCHOCH, P.; BINKLEY, D. Prescribed burning increased nitrogen availability in a mature lobloly pine stand. For. Ecol. Manag. Amesterdam, v.14, n.1, p.13-22, 1986.

SOARES, R.V. Incêndios Florestais - controle e uso do fogo. Curitiba: FUPEF, 213p, 1985. 
SOARES, R.V. Effects of a pine plantation prescribed burning on soil chemical properties in the savanna region of Minas Gerais state, Brasil. In: INTERNATIONAL CONFERENCE ON FOREST FIRE RESEARCH $(1,1990$, Coimbra). Proceedings. Coimbra: Universidade de Coimbra, 1990, p.C.06-C.09.

VEGA, J.A.; BARA, S.; GIL, M.C. Prescribed burning in pine stands for fire prevention in the N.W. of Spain: some results and effects. In: GOLDAMMER,J.G.(HRSg). DFG - Symposium "Feuerökologie". Albert Ludwigs - Universitat Freiburg i.Br. 1983, p.49-73.

WELLS, C.;CAMPBELL, R.E.; DE BANO, L.F.; LEWIS, C.E.; FREDRICKSEN, R.L.; FRANKLIN, E.C.; FROELICH, R.C.; DUNN, P.H.. Effects of fire on soil: a state of knowledge review. Gen. Tech. Rep., Washington, U.S.D.A. Forest Service, 34p. 1979 . 